\title{
Two-dimensional photonic crystals for GaN-based blue light emitters
}

\author{
Tomoyuki Yoshie ${ }^{* * * *}$ Chuan-Cheng Cheng ** and Axel Scherer ** \\ * Microelectronics Research Center, SANYO Electric Co., Ltd., 1-18-13, Hashiridani Hirakata, \\ Osaka 573-8534 JAPAN \\ ** Electrical Engineering, California Institute of Technology, MS200-36 Pasadena, CA 91125 USA
}

\section{Abstract}

We successfully fabricated two-dimensional photonic crystal structures for III-nitride light emitters by integrating electron-beam lithography, xenon/chlorine-based chemically assisted ion beam etching and a multilayer pattern transfer.

\section{Introduction}

Photonic crystals have recently attracted much attention because they can be used to control spontaneous emission in a small optical cavity[1,2]. By incorporating a defect in a photonic crystal, a high $\mathrm{Q}$ microresonator can be constructed. Low operating power and/or high modulation speed are expected from microcavity light emitters such as laser diodes (LDs) and light emitting diodes (LEDs) [3]. The high contrast of refractive index between semiconductors and air can produce photonic crystals in which light is efficiently reflected regardless a propagation direction. Though photonic crystal structure has been precisely fabricated in the long-wavelength compound semiconductor systems such as GaInAsP/InP and $\mathrm{AlGaAs} / \mathrm{GaAs}$ [4], crystals for the blue regime have not been fabricated because of the difficulty associated with finer pattern-transfer. Recently, the performance of $\mathrm{GaN}$ laser diodes has drastically progressed. Laser lifetime is reported to exceed 1000 hours with $\mathrm{cw}$ room temperature operation[4]. However, GaN-based microcavity lasers and vertical cavity surface emitting lasers[5] have so far not been successfully realized. To approach these devices, we report techniques to fabricate 2D-photonic crystals for blue III-nitride light emitters, which are expected to realize in-plane tight optical confinement of light in $\mathrm{GaN}$-based light emitters.

\section{Fabrication and Results}

Undoped GaN was used for this work. It was grown on c-face sapphire by metal organic vapor phase epitaxy (MOVPE). The total thickness of GaN epilayer is $2 \mu \mathrm{m}$. The epilayers were confirmed to have good optical quality by low temperature photoluminescence measurement.

To transfer patterns into GaN, we developed multilayer pattern transfer. We used high-resolution electron beam lithography for patterning 2D-photonic crystal structures. Our $30 \mathrm{kV}$ electron beam writing system has a field emission filament and a nominal spot size of $1 \mathrm{~nm}$. Polymethylmethacrylate (PMMA) was used as a positive electron beam resist. This allows us to define fine patterns such as photonic crystals for $\mathrm{GaN}$. As a mask, we deposited $\mathrm{Au} / \mathrm{Ni}$ on top of a $300 \mathrm{~nm} \mathrm{SiO}{ }_{2}$ deposition on the $\mathrm{GaN}$ epilayer. After electron beam lithography on PMMA, patterns were transferred into the $\mathrm{Au} / \mathrm{Ni}$ layer by argon ion milling and then into a $\mathrm{SiO}_{2}$ layer by $\mathrm{C}_{2} \mathrm{~F}_{6}$ reactive ion etching. Pattern-transfer into the $\mathrm{GaN}$ layer was conducted by $\mathrm{Cl}_{2}$-based chemically assisted ion beam etching (CAIBE). Xenon was used as a sputtering ion beam instead of argon to enhance the etching rate. GaN was etched at $220^{\circ} \mathrm{C}$ while locally supplying $\mathrm{Cl}_{2}$ in order to get vertical and smooth sidewall inside the etched trenches. The etching rate was $0.6 \mu \mathrm{m} / \mathrm{min}$. Our etching mask enables us to etch $1.5 \mu \mathrm{m}$ deep trenches. This is deep enough to confine light in-plane in active layers of InGaN/GaN/AlGaN LDs and LEDs. In InGaAs/GaAs microcavity LDs, trenches entering into the cladding layer under an active layer are deep enough to get a high $Q$ in photonic crystal microcavity[6].

Before fabricating the structures consisting of triangular lattice of circular trenches, we calculated 2D-photonic band structure of the infinitely periodical triangular lattice by using the plane-wave expansion method [7]. The photonic bandgap is between 0.35 and 0.50 of normalized frequency when the modal refractive index is 2.72 and the ratio of trench radius to trench pitch, r/a, is 0.4 . When we assume $410 \mathrm{~nm}$ as the emission wavelength, suitable pitch of trenches ranges between 140 and $200 \mathrm{~nm}$ in order that emission wavelength is in the photonic bandgap. Figure 1 shows scanning electron microscopy (SEM) images of photonic crystals with a range of different pitches. (0-7803-4223-2/98/\$10.00 1998 IEEE) 
Fig.1(a),(b) and (c) have pitches of trench: 150,170 and $190 \mathrm{~nm}$, respectively. A defect can be seen in photonic crystals in each image of Fig.1. The etched depth was confirmed to be over $1 \mu \mathrm{m}$.

The tequnique described here can be easily applied to InGaN/GaN/AlGaN LDs and LEDs. In the InGaAs/InGaAsP system, a membrane structure is appropriate because of the high refractive index of the InP substrate. In GaN-based light emitters, however, we can use sapphire substrate whose refractive index is 1.77 . Emission from the active region could be strongly localized in the defect when the $2 \mathrm{D}$ photonic crystal is applied to waveguide of the light in addition to such a vertically optical confinement structures.

\section{Conclusion}

We have demonstrated that 2D-photonic crystals for gallium nitride can be fabricated. The structures are enabled by integrating techniques of high resolution electron-beam lithography, $\mathrm{Cl}_{2}-$ based CAIBE with xenon ions and a multilayer pattern transfer.

\section{References}

[1] E. M. Purcell, Phys. Rev. 69,681 (1946)

[2] E. Yablonovitch, Phys. Rev. Lett. 58, 2059 (1987)

[3] T. Baba, IEEE J. of Selected Topics in Quantum Electronics 3, 808 (1997)

[4] J. O'Brien, O. Painter, C.C. Cheng, R. Lee, A. Scherer and A. Yariv, Electron. Lett. 32, 2243 (1996)

[5] S. Nakamura, M. Senoh, S. Nagahama, N. Iwasa, T. Yamada, T. Matsushita, H. Kiyouku, Y. Sugimoto, T. Kozaki, H. Umemoto, M.Sano and K. Chocho, Appl. Phys. Lett. 72, 211 (1998)

[6] B. D'Urso, O. Painter, J. O'Brien, T. Tombrello, A. Yariv and A. Scherer, J. Opt. Soc. Am. B $15,1155,(1998)$

[7] M.Plihal and A.A. Maradudin, Phys. Rev. B 44, 8565 (1991)

(a)
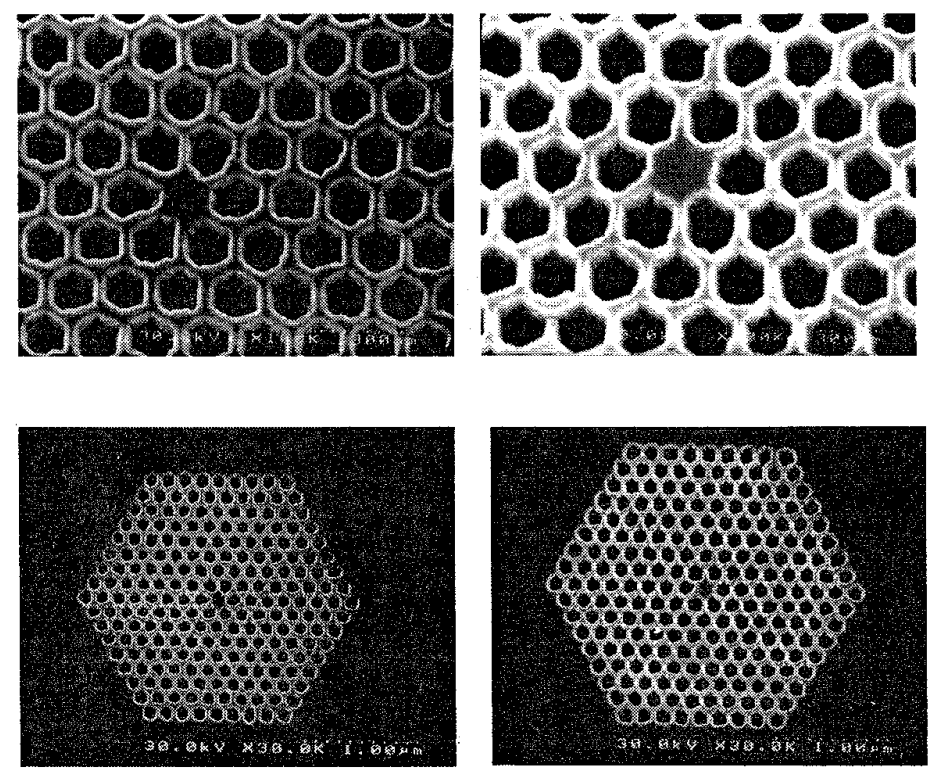

(c)

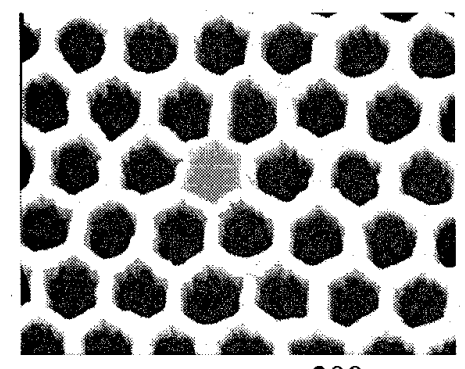

$300 \mathrm{~nm}$

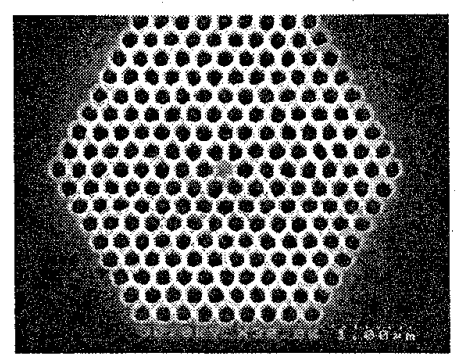

$1 \mu \mathrm{m}$

Fig.1 SEM images of photonic crystal structures consisting of triangular lattice in gallium nitride. Pitches of trench are (a) 150 , (b) 170 and (c) $190 \mathrm{~nm}$, respectively. 